\title{
Fotoprotección en la infancia
}

\author{
M. Valdivielso Ramos, C. Mauleón Fernández, E. Balbín Carrero, \\ P. de la Cueva Dobao, E. Chavarría Mur, JM. Hernanz Hermosa \\ Servicio de Dermatología, Hospital Infanta Leonor. Madrid. España.
}

Rev Pediatr Aten Primaria. 2009; I 1:3 13-24

José Manuel Hernanz Hermosa, jmhernanzh@salud.madrid.org

\section{Resumen}

Los niños son el grupo poblacional en el que más debe extremarse la fotoprotección. Son muy sensibles a los efectos negativos derivados de una exposición solar excesiva, y no son conscientes de este riesgo. Los niños desarrollan una actividad al aire libre mucho más importante que los adultos, y la exposición a la radiación ultravioleta y las quemaduras durante la infancia son un factor de riesgo fundamental para el desarrollo de cáncer cutáneo en la edad adulta.

Es por tanto de vital importancia proteger al niño de los efectos nocivos de la radiación ultravioleta. Las medidas de fotoprotección incluyen las conductas de evitación solar, la protección con ropa, gorros y gafas solares, y el uso de cremas fotoprotectoras.

Las campañas de educación solar deben desarrollarse en los hospitales, en los colegios, y en los medios de comunicación, de forma conjunta entre dermatólogos, pediatras, profesores y responsables gubernamentales.

Palabras clave: Fotoprotección, Infancia, Fotoprotectores solares.

\section{Abstract}

Children are the population group who most need photo protection. They are very sensitive to the negative consequences of an excessive sun exposure, and they aren't aware of the risk. Children have much more open air activity than adults, and the exposure to ultraviolet radiation and sun burns in childhood are a risk factor of the utmost importance in the development of skin cancer in adulthood.

It is therefore of vital relevance to protect the child from the deleterious effects of ultraviolet radiation.

The photo protection measures include sun avoidance, protection with cloths, caps and solar glasses, and the use of photo protection ointments.

Sun education campaigns should be implemented in hospitals, schools and communication media, and with dermatologists, pediatricians, teachers and political referents altogether.

Key words: Photo protection, Child, Sun protection ointments. 


\section{Introducción}

La mayoría de los efectos biológicos de la luz solar sobre la piel humana se deben a la radiación ultravioleta (UV), que se divide en tres tipos en función de la longitud de onda de la radiación: ultravioleta A, UVA (320-400 nm), que a su vez se divide en UVA1 (340-400 nm) y UVA2 (320-340 nm); ultravioleta B, UVB (290$320 \mathrm{~nm})$; y ultravioleta C, UVC (270-290 $\mathrm{nm})$.

Esta radiación es filtrada a través de diversas capas atmosféricas, que permiten el paso de un espectro de radiación que oscila entre la radiación ultravioleta B de 290 nm, y los infrarrojos lejanos de $4.000 \mathrm{~nm}$. La radiación UVC es filtrada por la capa de ozono en la estratosfera, ya que es muy tóxica para los seres vivos.

Cuanto más alto esté el sol en el cielo, mayor es la intensidad de la radiación UV. Diversos factores modifican la cantidad de radiación solar que llega a la tierra:

- La hora del día (la mayor radiación tiene lugar entre las 11 y las 16 horas).

- La estación del año (el verano es la de mayor intensidad).

- La latitud geográfica (mayor radiación cuanto más cerca del ecuador).
- La altitud (mayor radiación cuanto mayor sea la altitud).

- Las nubes y la polución disminuyen la cantidad de radiación.

\section{Peculiaridades de la piel del neonato}

En el momento del nacimiento la piel no ha completado su desarrollo definitivo y difiere en algunos parámetros de la del adulto. Se produce un proceso de maduración de la piel, que incluye un descenso en el pH de la superficie cutánea; un aumento de la descamación cutánea, sobre todo a nivel facial; y un aumento de la hidratación del estrato córneo.

La piel del neonato tiene una alta perfusión sanguínea, una gran hidratación del estrado córneo que contribuye a aumentar la permeabilidad del mismo, y una mayor superficie cutánea relativa, lo que facilita la absorción de sustancias por vía percutánea.

\section{Efectos de la fotoexposición}

Los efectos dañinos de la radiación UV son acumulativos e irreversibles. Unos efectos de la radiación solar son inmediatos, como la aparición de eritema, pigmentación o quemaduras solares. Otros efectos acontecen de forma tardía, como el envejecimiento cutáneo o el aumento de riesgo de carcinogénesis. 
La radiación ultravioleta también es responsable de la aparición de trastornos de fotosensibilidad y de la supresión del sistema inmunológico, en el que participan tanto los UVB como los UVA'. Existen varias enfermedades que se agravan con la exposición solar como el lupus, la porfiria o el xeroderma pigmentoso, y que característicamente afectan a los niños.

La exposición solar intensa durante las dos primeras décadas de vida se ha relacionado especialmente con el fotoenvejecimiento cutáneo y la formación de cáncer de piel. El cáncer cutáneo es el tipo de cáncer más frecuente en el mundo y el melanoma el que más rápidamente está aumentando, según las últimas publicaciones de la Organización Mundial de la Salud (OMS)2.

La radiación UV, y, de manera especial, la exposición temprana e intensa durante la infancia es el principal factor de riesgo para el cáncer de piel, especialmente el carcinoma basocelular, el carcinoma espinocelular, y el melanoma ${ }^{3}$. Se ha demostrado una intensa correlación entre las quemaduras en la infancia y el riesgo de melanoma ${ }^{4,5}$. También se ha documentado una relación entre el melanoma y la exposición intermitente a la radiación solar, que a su vez se relaciona significativamente con el aumento de las quemaduras solares ${ }^{6}$.
Existe también una asociación entre la exposición solar en la infancia y el número de nevus melanocíticos adquiridos, cuyo número elevado también se considera un factor de riesgo de melanoma.

La disminución de la exposición a la radiación UV en la infancia podría potencialmente disminuir de forma significativa la incidencia del cáncer de piel tanto en la infancia como en la edad adulta.

La piel juega un papel fundamental en la biosíntesis de vitamina $\mathrm{D}$. Para que esta función se produzca con normalidad se necesitan dosis pequeñas de radiación UV. La asociación de una dieta equilibrada y de una exposición solar mínima en un país con abundante radiación solar como España, es suficiente para cubrir las necesidades en la infancia ${ }^{7}$.

\section{Fotoprotección infantil}

La fotoprotección incluye todas aquellas medidas que nos permiten protegernos de las radiaciones solares. La necesidad de protegerse de la radiación UV se antoja como urgente, y debe comenzar en la infancia.

Aunque las medidas de fotoprotección son aconsejables en todas las edades, estas deben ser más intensas en la población infantil y juvenil, ya que los niños son más susceptibles que los adultos a las radiaciones UV. De hecho algunos traba- 
jos establecen que entre un 50-80\% del daño inducido por la exposición solar que un individuo recibe a lo largo de toda la vida se realiza durante la infancia y la adolescencia ${ }^{8}$.

Otra consideración importante en el caso de los niños, es que mientras son muy pequeños, son sus padres los que tienen que responsabilizarse de su fotoprotección, y de fomentar estos hábitos saludables en los niños con su propio ejemplo. En un estudio llevado a cabo en los Estados Unidos durante el verano de 1997, los autores demostraron que un $13 \%$ de los niños habían sufrido quemaduras en la semana previa.

La FDA no recomienda la aplicación de fotoprotectores en niños menores de 6 meses, evitando de esta forma hipotéticos problemas de toxicidad secundarios a la mayor absorción y superficie relativa de la piel del neonato. Estos niños tampoco deben exponerse directamente al sol sin ropas protectoras, ya que tienen menor capacidad de producción de melanina y de sudoración, por lo que son especialmente susceptibles a las quemaduras solares y al calor. También se recomienda limitar las exposiciones solares en niños por debajo de los 3 años de edad.

La fotoprotección exógena incluye todas aquellas medidas que podemos instaurar de forma externa para proteger a la piel de la agresión producida por las radiaciones solares. Ya hemos señalado que en el caso de los niños más pequeños son los padres quienes tienen que preocuparse de realizar todas estas medidas de fotoprotección, y de ir afianzando estas conductas con el paso del tiempo, para que los niños las vayan desarrollando de forma progresiva e ininterrumpida, a medida que va aumentando su autonomía.

La medida más importante consiste en evitar o disminuir el tiempo en el que los niños estén expuestos a la radiación solar. Durante la infancia se realizan un mayor número de actividades al aire libre, por lo que con frecuencia se produce una exposición solar prolongada. Se calcula que los niños reciben el triple de radiación solar que los adultos. Debe aconsejarse la disminución de las actividades al aire libre en los períodos centrales del día, fomentar el uso de lugares sombreados, o evitar la exposición solar intencionada (incluyendo las cabinas de bronceado) con el fin de conseguir un bronceado perfecto. Las sombras y los árboles reducen la radiación UV directa, pero no la indirecta emitida por las superficies circundantes, como el agua, la arena o la nieve.

Ya que casi la mitad del año los niños lo pasan en las escuelas, es su responsa- 
bilidad fomentar el uso de las sombras, proporcionar adecuadas sombras en los patios de los colegios, y realizar las clases de educación física al aire libre en los períodos del día con menor radiación solar. Se ha estimado en un $47 \%$ la exposición solar diaria que reciben los niños mientras se encuentran al aire libre en los descansos en los colegios ${ }^{9}$. La regla de la sombra puede ayudarnos a saber cuándo debemos poner más empeño en evitar el sol, ya que nos avisa de que el sol es más intenso y las quemaduras más probables. Esto ocurre cuando las sombras son más cortas en relación a la altura del niño, y el riesgo es menor cuando el tamaño de la sombra sea mayor ${ }^{9,10}$.

Otra medida muy importante es la limitación del uso intencional de la exposición a la radiación UV, ya sea al aire libre, o en las cabinas de bronceado. Entre un $11-37 \%$ de los adolescentes estadounidenses han utilizado las cabinas de bronceado ${ }^{9}$. Parece necesaria una regulación más estricta del uso de las mismas por los más jóvenes, mediante una legislación oficial menos permisiva. También sería interesante la disminución de la edad en la que estos jóvenes pueden utilizar las cabinas sin el consentimiento de sus padres.

Si vamos a estar expuestos al sol, es conveniente cubrir la mayor parte del cuerpo posible de las radiaciones solares, mediante la utilización de ropa, sombreros y gafas fotoprotectoras. El uso de gorras de protección es especialmente importante en los niños. Las gafas de sol protegen los ojos y las regiones perioculares.

Existen tejidos especiales que llevan materiales con protección solar. El factor de protección ultravioleta (FPU) es la medida de transmisión de UV a través del tejido. Posiblemente en el futuro todas las ropas lleven su FPU en la etiqueta, al igual que las cremas fotoprotectoras llevan el FPS (factor de protección solar) ${ }^{11}$. Se afecta por muchos factores, como el tipo de material, el color, el peso, la porosidad, el grosor, la elasticidad, la hidratación y los procedimientos de acabado de fábrica ${ }^{12-14}$. La protección es mayor en los colores más oscuros, en los tejidos tratados con detergentes que contengan un absorbente de UV, cuanto menores sean los espacios entre los hilos, y cuanto mayor sea el peso y el grosor del tejido. El nailon, la lana, la seda y el poliéster tienen mayor factor de protección que el algodón, la viscosa, el rayón y el lino ${ }^{14,15}$.

Algunos autores han demostrado que llevar ropas protectoras puede disminuir el número de nevus melanocítico adquirido ${ }^{16}$. 


\section{Fotoprotectores}

Los fotoprotectores son aquellas sustancias que absorben y filtran la radiación UV (evitando su penetración hacia epidermis y dermis), dispersan y reflejan las radiaciones. Algunos fotoprotectores actuales también tienen capacidad de inactivar los radicales libres que se producen, $y$, por lo tanto, de prevenir o de reparar los daños inducidos por las radiaciones solares ${ }^{15}$. Su utilización es esencial, ya que constituyen una medida fundamental de protección frente a la radiación ultravioleta. Se ha demostrado que tienen efectos positivos en disminuir los signos del envejecimiento y la incidencia del cáncer de piel ${ }^{17}$.

Hay que potenciar el uso correcto del fotoprotector en todos los períodos de vida, pero en la infancia es aún más importante. Es necesario que tanto pediatras como dermatólogos colaboren de manera conjunta en fomentar el uso de fotoprotectores y de otras barreras de protección solar. Parece que el grupo de edad en el que con menor probabilidad se emplean los fotoprotectores solares es el de 17 a 24 años $^{18}$. No hay diferencias en los niños con respecto a los adultos en cuanto a la penetración de los fotoprotectores tópicos, ni en la capacidad de irritación de la piel frente a sustancias externas. La gran diferencia en cuanto a la aplicación de sustancias tópicas es por la mayor proporción de superficie corporal de los niños, por lo que la exposición sistémica a sustancias de aplicación tópica es superior en los niños con respecto a los adultos ${ }^{15}$.

Son los padres los que deben facilitar el uso de los fotoprotectores en los niños más pequeños, y utilizarlos con generosidad tanto en ellos como en sí mismos, dando ejemplo de conductas fotoprotectoras. El uso de los fotoprotectores en los niños no es el adecuado, y se ha demostrado que tan solo el $53 \%$ de los adultos refieren que sus hijos siempre o con mucha frecuencia se aplican el fotoprotector, y un $44 \%$ que lo aplican repetidamente ${ }^{8,9}$.

Como medidas generales necesarias para conseguir una mayor efectividad del fotoprotector, destacaremos:

- El fotoprotector debe aplicarse generosamente de 15 a 30 minutos antes de la exposición solar.

- Debe reaplicarse en zonas fotoexpuestas cada 1-2 horas, o tras actividades físicas intensas que puedan eliminarlo de la piel como nadar, sudar excesivamente, o limpiarse con la toalla. La reaplicación del producto incrementa de 2 a 3 veces la protección frente al sol'.

Esto último es necesario aunque el filtro sea impermeable o resistente al agua 
(waterproof, water-resistant). Se define como resistente al agua (water-resistant) cuando el FPS se mantiene después de 40 minutos de inmersión en agua, e impermeable (waterproof) cuando es efectivo después de una inmersión de 80 minutos ${ }^{3}$.

El factor de protección solar (FPS) es un índice definido como el cociente del tiempo de exposición de radiación UV requerido para producir un eritema (dosis eritematógena mínima) en la piel protegida por el producto y sin él, a las 24 horas de la irradiación ${ }^{19}$. Se mide in vivo según las recomendaciones de la FDA aplicando $2 \mathrm{mg} / \mathrm{cm}^{2}$ sobre la piel de la espalda, o $30 \mathrm{~g}$ en la totalidad del cuerpo, 30 minutos antes de la irradiación. Aunque en las mediciones se emplea esa cantidad, en la práctica se emplea mucho menos, entre $0,5-1,3 \mathrm{mg} / \mathrm{cm}^{2}$, por lo que en las condiciones reales el FPS alcanzado es considerablemente menor que el esperado, y en muchos casos corresponde al 20-50\% del indicado en la etiqueta del producto 9 .

El FPS es una medida reconocida; sobre todo la protección frente a UVB, que es la radiación responsable fundamentalmente del eritema solar y de las quemaduras. No hay un método estandarizado para medir la eficacia del bloqueo frente a UVA, aunque se utilizan varios test in vivo e in vitro para medirlo, como el de la pigmentación inmediata, el de la pigmentación persistente $\mathrm{y} / \mathrm{o}$ el test del factor de protección ${ }^{20}$. Cuanto mayor capacidad de absorción de la radiación UVA tenga el producto, mayor prevención del fotoenvejecimiento cutáneo aporta.

En la Unión Europea el método más usado para la medición del FPS para la radiación UVB es el COLIPA. La protección frente a la radiación UVB, como se refleja en el FPS es la principal propiedad que determina la potencia de un fotoprotector. El sistema de numeración de los FPS no es lineal, en los factores bajos de protección la actividad real del producto se incrementa considerablemente, y en los valores altos los aumentos de FPS representan incrementos mínimos de reducción.

\section{Tipos de fotoprotectores}

Los fotoprotectores están formados por la mezcla de unos principios activos y de unos excipientes que vehiculizan dichas sustancias. Las formulaciones actuales incluyen cremas, geles, espráis, espumas y lociones. Las diferencias entre todas ellas determinan la permanencia del producto en la piel, $y$, sobre todo, la cosmética del producto. Las lociones y los espráis son más sencillos de utilizar en los niños, ya que permiten una aplicación 
más rápida. Para que el acto de aplicar la crema se convierta en algo más divertido, algunas casas comerciales incluyen cremas de colores en sus formulaciones.

Los principios activos se dividen en filtros físicos o químicos, con importantes peculiaridades entre ambos.

Los fotoprotectores químicos u orgánicos absorben la energía trasportada por los fotones de las radiaciones UV, por lo que pueden considerarse como cromóforos exógenos ${ }^{15}$. Son muy variados y tienen mecanismos de acción diferentes, transformando en general la energía lumínica en energía térmica. Algunos absorben sobre todo la radiación UVB como el ácido paraaminobenzoico (PABA, cuyo uso está limitado por las frecuentes reacciones adversas cutáneas que producen), los cinamatos, los salicilatos y el octocrileno. Otros absorben UVA como las benzofenonas, las antralinas, la avobenzona y el ácido tereftalideno-dialcanfor sulfónico (Mexoryl SX). Algunos absorben ambas radiaciones como el Tinosorb $\mathrm{M} \mathrm{o} \mathrm{Ti-}$ nosorb $S$ (metoxifeniltriazina). En general estos filtros son incoloros y cosméticamente muy aceptables, aunque presentan un mayor riesgo de reacciones de contacto que las pantallas minerales.

Los fotoprotectores físicos, inorgánicos o pantallas minerales, actúan como una barrera física, absorbiendo y disper- sando la radiación UV, así como la luz visible y los infrarrojos. Se consideran muy seguros, y son los que se utilizan en aquellos fotoprotectores dirigidos de forma específica a la infancia. Contienen pequeñas partículas de 180 a 250 nm de dióxido de titanio, óxido de zinc, óxido de hierro, óxido de magnesio, mica, talco, caolín o calamina ${ }^{15}$. Estos minerales bloquean la radiación gracias a sus propiedades físicas de índice de refringencia, y el tamaño y el espesor de las partículas. Estos filtros no son irritantes ni sensibilizantes, ya que se trata de polvos inertes, por lo que se usan como primera elección en pacientes con historia de alergia a los fotoprotectores. En particular el óxido de zinc y el dióxido de titanio no penetran más allá del estrato córneo, con lo que se impide cualquier posibilidad de absorción sistémica ${ }^{10}$. Al aplicarlos la piel se torna blanquecina, por lo que no son muy aceptados por los pacientes. La cosmética de estos agentes se ha mejorado enormemente con las nuevas formulaciones micronizadas, que han eliminado esa apariencia de "pasta blanca" en la piel.

En algunos preparados se añaden agentes antioxidantes, que actúan directamente en la piel alcanzando concentraciones suficientes y eliminando los radicales libres de oxígeno generados por la radiación UV. Entre ellos destacan la vi- 
tamina $C$ y $E$, los carotenoides, polifenoles del té verde, o los flavonoides ${ }^{21}$.

En general, en los niños más pequeños se aboga fundamentalmente por la seguridad, y son preferidos los filtros físicos, que garantizan una menor absorción de ingredientes tóxicos, y de aparición de reacciones irritativas o alérgicas. Los estudios clínicos realizados para evaluar la absorción sistémica de los productos químicos utilizados en los fotoprotectores de niños y de adultos, han puesto de manifiesto unas tasas de penetración similares en ambos grupos. Sin embargo, parece prudente evitar aquellos productos que contengan PABA y benzofenonas como la oxibenzona. No parece que la piel de los niños sea más susceptible a irritarse por la aplicación de preparados tópicos que la de los adultos.

Resumiendo, los fotoprotectores deben reunir unas características que incluyan:

- Amplio espectro.

- Fotoestabilidad.

- Alta sustantividad.

- Cosmética agradable.

- Aplicación fácil.

- No irritante.

- No manche.

- Precio aceptable.

En los últimos años se ha desarrollado el uso de fotoprotectores sistémicos, que se administran por vía oral. Teóricamente tienen como ventajas la protección de la totalidad de la piel, y que su validez no está sujeta a la forma de aplicación. Entre ellos destacaremos los $\beta$-carotenos, Polipodium leucotomos, combinaciones de antioxidantes orales como la vitamina $C$ y $E$, y los polifenoles del té verde. Los estudios aportan conclusiones contradictorias en cuanto a su eficacia, y no hay estudios de eficacia en niños.

\section{Recomendaciones prácticas}

Como ya hemos señalado, en los niños menores de 6 meses debe evitarse la exposición solar directa $y$, también, el uso de los fotoprotectores.

En los niños mayores de 6 meses deben aplicarse todas las medidas de fotoprotección. Las estrategias de fotoprotección de acuerdo con las guías nacionales e internacionales incluyen:

- Evitar la exposición solar, especialmente entre las 11 y las 16 horas.

- Cubrirse con ropas y gorros adecuados.

- Llevar gafas con protección solar.

- Uso de fotoprotectores.

- Protegerse también en días nublados, porque la radiación UV puede atravesar las nubes.

- Protegerse del sol aunque la piel esté bronceada, porque puede quemarse. 
En cuanto a las características de los fotoprotectores:

- Deben tener un FPS mayor o igual a 25.

- Amplio espectro (deben cubrir la radiación UVA y UVB).

- Resistentes al agua y al frotamiento.

- Texturas de buena cosmeticidad.

En cuanto a la aplicación de los fotoprotectores:

- Deben aplicarse generosamente 15-30 minutos antes de la exposición solar, para mejorar su permanencia en la piel.

- Reaplicarlos en las zonas fotoexpuestas de 15 a 30 minutos después de comenzada la exposición solar, y posteriormente cada 2 horas y/o al bañarse, frotarse con la toalla, o sudar excesivamente.

- Antes de usarlos conviene agitarlos bien para mezclar las partículas.

- Aplicar suficiente protector.

- Aplicarlo en todas las zonas expuestas al sol, incluidos los pabellones auriculares, hombros y cara posterior de rodillas y piernas. Tener cuidado con la aplicación cerca de los ojos, ya que puede provocar escozor.

Diversos estudios han demostrado diferencias entre la eficacia teórica de un fotoprotector (la medida en el laborato- rio) con la real. Esto puede ocurrir por diferencias en la cantidad, modo de aplicación, variabilidad del espectro de radiación UV dependiendo de la hora o la latitud, y/o por la evolución fotoquímica de los filtros en el transcurso de la exposición solar ${ }^{15}$.

\section{Educación solar}

Todos los dermatólogos sabemos qué importante es la fotoprotección para prevenir la formación de cáncer cutáneo. Sin embargo, llama la atención la escasa información que posee la población general, a pesar de las múltiples campañas informativas. La importancia de la divulgación en la comunidad de los conocimientos sobre las medidas fotoprotectoras, y la promoción de la fotoprotección solar en los niños y sus padres es parte de una estrategia global que persigue la obtención de cambios de comportamiento positivos frente al sol, y la disminución de la incidencia del cáncer cutáneo en el futuro. Debe favorecerse el uso no solo de las cremas fotoprotectoras, sino de todos aquellos métodos que reducen la exposición solar.

La actuación sinérgica de dermatólogos y pediatras es esencial en esta labor educacional, conjuntamente con las escuelas y los programas comunitarios, so- 
bre todo en aquellos países más soleados, como es el caso de España ${ }^{22}$.

Se han desarrollado múltiples campañas educacionales en diferentes países, incluida España, destinadas a la adquisición de hábitos solares saludables, y a la ampliación del conocimiento de los efectos nocivos de la radiación UV en la población infanti ${ }^{22-24}$. Es importante que la información se presente de forma positiva, de manera que se permita a las personas disfrutar del sol con seguridad, pero siendo al mismo tiempo conscientes de la necesidad de evitar la sobreexposición. La mayoría de estas intervenciones tiene como dianas a los niños. Se ha sugerido que las edades de 9 a 12 años son los años críticos, donde los niños comienzan a ser independientes de sus padres en la protección solar, y por lo tanto, cuando sus comportamientos de fotoprotección comienzan a fallar ${ }^{25}$.

Todas las prácticas sanas que evitan o minimizan la exposición solar, que favo- recen el uso de ropa protectora, gorros, gafas y cremas fotoprotectoras, que evitan la formación de quemaduras solares, o que disminuyen el uso de las cabinas de bronceado, deben comenzarse lo antes posible en los niños, para que en estos se convierta en algo rutinario. Está demostrado que cuanto menor es la edad en la que se adquieren los comportamientos saludables, con mayor probabilidad se perpetúan en la edad adulta ${ }^{23}$.

Es fundamental realizar campañas de educación sobre los comportamientos frente al sol en los hospitales, de forma conjunta entre pediatras y dermatólogos, y en las escuelas, donde tanto profesores como médicos puedan facilitar el acceso a la información a los más jóvenes. Los responsables de la salud pública deberían colaborar también en hacer llegar este mensaje, mediante campañas divulgativas en los medios de comunicación, insistiendo en que la protección solar es un problema de salud pública.

\section{Bibliografía}

1. Moyal D, Fourtanier A. Broad spectrum sunscreens provide better protection from solar ultraviolet-simulated radiation and natural sunlight induced immunosuppression in human beings. J Am Acad Dermatol. 2008;58 (5):s149s154.
2. Abdulla FR, Feldman SR, Willoford $P M$, Krowchuk D, Kaur M. Tanning and skin cancer. Pediatr Dermatol. 2005;22:501-12.

3. Mac Neal RJ, Dinulos J. Update on sun protection and tanning in children. Curr Opin Pediatr. 2007;19:425-9.

4. Devesa SS, Silverman BT, Young UL. Cancer incidence and mortality trends among whites in 
the US, 1947-1984. J Natl Cancer Inst. 1987;79; 701-77.

5. Oliveira SA, Saraiya M, Geller AC. Sun exposure and risk of melanoma. Arch Dis Child. 2006;91:131-8.

6. Elwood JM, Jopson J. Melanoma and sun exposure: an overview of published studies. Int J Cancer. 1997;73:198-203.

7. Holick MF. Sunlight, UV-radiation, vitamin $D$ and skin cancer: how much sunlight do we need? Adv Exp Med Biol. 2008;624:1-15.

8. Robinson JK, Rigel DS, Amonette RA. Summertime sun protection used by adults for their children. J Am Acad Dermatol. 2000;42:746-53.

9. Eide MJ, Weinstock MA. Public health challenges in sun protection. Dermatol Clin. 2006; 24:119-24.

10. Jones Caballero $M$, Fernández Peñas $P$. Puesta al día en fotoprotección: tipos, indicaciones, novedades y controversias. Dermatol Práctica. 2008;16:4-16.

11. Morison WL. Photoprotection by clothing. Dermatol Ther. 2003;16:16-22.

12. Almahroos $M$, Kurban A. Sun protection for children and adolescents. Clin Dermatology. 2003;21:311-4.

13. Gambichler T, Avermaete A, Bader A. UItraviolet protection by summer textiles. Ultraviolet transmission measurements verified by determination of minimal erythema dose with solar-simulated radiation. Br J Dermatol. 2001; 144: 484-9.

14. Hoffman K, Laperre J, Avermaete A. Defined UV protection by apparel textiles. Arch Dermatol. 2001;137:1089-94.

15. Gilaberte Y, Coscojuela C, Sáenz de Santamaría MC, González S. Fotoprotección. Actas Dermosifiliogr. 2003;94:271-93.
16. Autier P, Dore JF, Shifflers E. Melanoma and the use of suscreens: an EORTC case-control study in Germany, Belgium and France. The EORTC Melanoma Cooperative Group. Int J Cancer. 1995;61:749-55.

17. Palm MD, O'Donoghue MN. Update on photoprotection. Dermatol Therapy. 2007;20: 360-76.

18. Schofield PE, Freeman JL, Dixon HG. Trends in sun protection behaviour among Australian young adults. Aust N Z J Public Health. 2001;25:62-5.

19. Schultze R. Einige versuche und bemerkungen zum problem der handelsublichen lichtschutzmittel. Parfüm Kosmet. 1956;37:310-65.

20. Lautenschlager $S$, Wulf HC, Pittelkow MR. Photoprotection. Lancet. 2007;370:528-37.

21. Eberlein-Konig B, Ring J. Relevance of vitamins $C$ and $E$ in cutaneous photoprotection. J Cosmet Dermatol. 2005;4:4-9.

22. Dietrich $A J$, Olson $A L$, Sox $C H$, Stevens $M$, Tosteson TD, Ahles T, et al. A community-based randomized trial encouraging sun protection for children. Pediatrics. 1998;102:e64.

23. Gilaberte Y, Alonso JP, Teruel MP. Evaluation of a health promotion intervention for skin cancer prevention in Spain: the Solsano program. Health Promot Int. 2008;23:209-19.

24. Saraiya M, Glanz K, Briss P. Interventions to prevent skin cancer by reducing exposure to ultraviolet radiation. Am J Preventive Med. 2004;27:422-66.

25. Benjes LS, Brooks DR, Zhang Z. Changing patterns of sun protection between the first and second summers for very young children. Arch Dermatol. 2004;140:925-30. 